\title{
An Improved Model-Free Predictive Current Control with Advanced Current Gradient Updating Mechanism
}

\author{
Chenwei Ma, Student Member, IEEE, Huayu Li, Student Member, IEEE, Xuliang Yao, Zhenbin \\ Zhang, Senior Member, IEEE, and Frederik De Belie, Member, IEEE
}

\begin{abstract}
Model-based predictive control that highly depends on system parameters has been widely investigated. In contrast, model-free predictive current control (MFPCC) can be performed based on input or output measured data, rather than on any system model information, hence eliminating the influence of parameter uncertainties. In such a strategy, the current gradients due to each of the possible voltage vectors are stored and used to predict future currents. Current gradient knowledge therefore provides a significant foundation for MFPCC. In conventional MFPCC, however, the stagnation existing in the current gradient update always impacts the reliability of current gradients and further worsens the control performance. In this paper, an improved MFPCC with an advanced current gradient updating mechanism is proposed. In the proposed strategy, to guarantee the reliability of the current gradients, two contiguous measured current gradients due to the applied voltage vectors are used to estimate the current gradients for all the possible voltage vectors. This simple method takes advantage of the mathematical relationships between the voltage vectors. In this way, all the current gradients can be obtained within one control period, effectively reducing the stagnation effect in conventional MFPCC. The proposed MFPCC scheme is evaluated on a permanent magnet synchronous machine (PMSM) drive setup to demonstrate its effectiveness.
\end{abstract}

Index Terms-Permanent magnet synchronous motor (PMSM), model predictive control (MPC), model-free.

\section{INTRODUCTION}

$\mathrm{D}$ UE to the rapid development and various applications of electric machine drives, different control strategies have been widely investigated, such as field-oriented control (FOC)

C. Ma is with the College of Intelligent Systems Science and Engineering, Harbin Engineering University, Harbin 150001, China, is with the Department of Electrical Energy, Metals, Mechanical Constructions \& Systems, Ghent University, Ghent 9000, Belgium, and also with Flanders Make-EEDT (the strategic research center for the manufacturing industry), Leuven 3001, Belgium (e-mail: Chenwei.ma@ugent.be).

$\mathrm{H}$. Li and F. De Belie are with the Department of Electrical Energy, Metals, Mechanical Constructions \& Systems, Ghent University, Ghent 9000, Belgium, and also with Flanders Make-EEDT (the strategic research center for the manufacturing industry), Leuven 3001, Belgium (e-mail: Huayu.li@ugent.be; Frederik.DeBelie@UGent.be).

$X$. Yao is with the College of Intelligent Systems Science and Engineering, Harbin Engineering University, Harbin 150001, China (e-mail: yaoxuliang@hrbeu.edu.cn).

Z. Zhang is with the School of Electrical Engineering, Shandong University, Jinan 250061, China (e-mail: zbz@sdu.edu.cn). and direct torque control (DTC) [1-3]. In recent years, model predictive control (MPC), that can predict the system behaviors and include nonlinearities and multiple restraints, has received wide attention [4-6].

Due to the discrete nature of motor drive systems, the number of the working states is limited. Thus, the motor behaviors under the possible switching states of the inverter can be predicted and an optimal voltage vector can be decided upon based on a predefined cost function that includes the control objectives. For motor driving systems, this finite control set model predictive control (FCS-MPC), with an intuitive concept, is considered an alternative control method to the traditional ones [7].

A major drawback of a conventional FCS-MPC strategy is the steady-state performance because a single voltage vector is applied in each control period, which leads to high steady-state ripples and bad THD performance. Therefore, some two-vector-based MPCs [8-9], and control-set-extended MPCs [10-11], have been introduced. The main idea behind these strategies is to involve more potential control actions to improve the steady-state performance. Another disadvantage of an MPC is that all the predictions are based on a discrete system model, which means the control performance is largely dependent on the accuracy of the system parameters.

In [12], the parameter sensitivity of the model-based predictive current control (MBPCC) for a permanent magnet synchronous machine (PMSM) is analyzed. It concludes that the current predictions can be significantly affected by incorrect motor inductances. In contrast, the influence of resistance inaccuracy on the control performance is relatively weak. Some robust MBPCC strategies with inductance disturbance observers have therefore been proposed for the purpose of online parameter identification [12-14]. However, although the inductance parameter mismatch can be reduced by employing observers, this introduction can increase the complexities of the control strategies. Instead of using an observer, an alternative solution that utilizes a newly designed cost function in proportional-integral (PI) form is proposed in [15] to improve the robustness of MBPCC. In such a strategy, the past current tracking errors and future tracking errors are included in the cost function to be evaluated. A reduction of the steady-state error caused by parameter mismatches can be then achieved. However, the PI-based controller involves additional coefficients to be tuned, although a selective optimization method is introduced in such a strategy to facilitate the design of integral coefficients. In addition, the coverage problem is also needed to be considered for a PI-based controller.

On the other hand, a model-free predictive current control (MFPCC) is proposed in [16] to eliminate the influence of 
parameter uncertainties. Here, an MFPCC predicts the optimal control input based on the present and previous input/output data without the need of a system model, which is required in a conventional MBPCC [17]. In [16], the traditional model based prediction is abandoned. Instead, the current gradients caused by each voltage vector over a control period are stored in two look-up tables (LUTs) for the d-axis and q-axis, respectively. Then, assuming that a subsequent current gradient is equal to the previous one due to the same voltage vector, the predictions of the stator current can be approximately obtained based on the measured currents and the stored current gradients. In this way, only measured data are required, thereby skipping the motor parameters and system nonlinearities. Because the accuracy of the current prediction depends on the information in the LUTs, which must be updated online, the reliability of the LUTs is quite important for this approach. In [16], however, because only the current gradient caused by the applied voltage vector can be updated in one control period, the other elements in the LUT will be stagnant. Even worse, if a voltage vector is not applied over many periods, a long stagnation can therefore result in unreliable predictions and even impact the system stability.

The same problem can also occur in the robust predictive current control presented in [18], which includes a prediction error correction. To lessen the impact of this stagnation effect in the MFPCC, a minimum updating frequency is defined in [19], such that a voltage vector must be applied in the next control period if it has not been selected in a predefined number of past periods. However, the updating frequency for the LUTs is still not sufficient with this method. Furthermore, applying a non-optimal voltage vector frequently can cause a negative impact on the control performance. Therefore, such a conventional MFPCC requires further developments and improvements.

To enhance the reliability of the LUTs, an indirect LUT reconstruction approach is proposed in [20] and [21]. In such strategies, the current gradients caused by the last three applied voltage vectors are used to estimate the current gradients for the remaining vectors. However, this update can be fulfilled only when the three successive voltage vectors are different from each other, such that the LUT update can be corrupted if only two voltage vectors are activated for many periods, which often occurs in conventional MBPCC and MFPCC. In addition, to reconstruct the LUTs, all the possible vector sequences (up to 210) need to be considered. This means additional efforts are required to reduce the computational burden.

In this paper, an improved MFPCC is proposed for PMSM by introducing an advanced current gradient updating mechanism. Such a MFPCC does not require a mathematical model, and instead employs information about the current gradients to predict future currents. In this way, the impact of motor parameter mismatches can be totally avoided. The accuracy of the current gradients can be considered as key to this strategy. In this paper, the relationship between the current gradient and the applied voltage vector is first analyzed. The current gradients can be then derived for all the voltage vectors in one control period by further analyzing the relationships between the applied voltage vector and the other vectors. As a result, the stagnation problem that exists in the conventional MFPCC can be effectively solved by keeping all the current gradient information up to date.

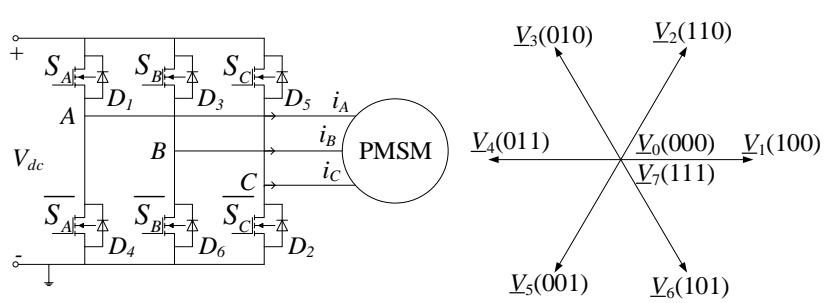

Fig. 1. Two level VSI and the candidate voltage vectors.

Compared to [20] and [21], which require three successive current gradients, the proposed mechanism utilizes samples from only two control periods to update all the LUT elements, significantly reducing the stagnation caused by having only two voltage vectors applied for long intervals. In addition, in contrast to [20] and [21], which involve considering all the possible vector sequences and taking action according to a predefined LUT, here, the current gradient update is performed based on simple and intuitive calculations. With reliable information about current gradients, the future currents can be accurately predicted and improved control performance can be therefore achieved. This strategy can prove valuable under conditions where the motor parameters cannot be properly given. In addition, in contrast to conventional MFPCCs, the proposed approach can potentially be extended to multiple-vector-based MFPCCs to improve the steady-state performance. Experimental results verify the effectiveness of the proposed strategy.

\section{Model-Based Predictive Current Control}

\section{A. PMSM Model}

In this paper, interior PMSM (IPMSM) is studied in rotary reference (d-q) frame. The model can be expressed as follows:

$$
\begin{aligned}
& v_{d}=R_{s} i_{d}+L_{d} \frac{d i_{d}}{d t}-L_{q} \omega_{r} i_{q} \\
& v_{q}=R_{s} i_{q}+L_{q} \frac{d i_{q}}{d t}+L_{d} \omega_{r} i_{d}+\omega_{r} \varphi_{f}
\end{aligned}
$$

where $v_{d}$ and $v_{q}$ are the $\mathrm{d}$-axis and q-axis stator voltage, respectively; $i_{d}$ and $i_{q}$ are the d-axis and q-axis stator current, respectively; $R_{s}$ is the stator resistance; $L_{d}$ and $L_{q}$ are the $\mathrm{d}$-axis and q-axis inductance, respectively; $\omega_{r}$ is the electrical rotor speed; and $\varphi_{f}$ is the permanent magnet (PM) flux linkage.

As shown in Fig. 1, a traditional two-level voltage source inverter (VSI) is employed to drive the PMSM. The candidate voltage vectors can be described as follow:

$$
\underline{V}_{j}=\frac{2}{3} V_{d c}\left(S_{A}+e^{i 2 \pi / 3} S_{B}+e^{i 4 \pi / 3} S_{C}\right)
$$

where $\underline{V}_{j}(j=0, \ldots, 7)$ are the candidate voltage vectors and $S_{A}, S_{B}, S_{C}$ are the switching states of the three inverter arms.

\section{B.MBPCC Strategy}

Based on the model (1), the stator current at the start of the next control period $(\mathrm{k}+1)$ can be deduced as 


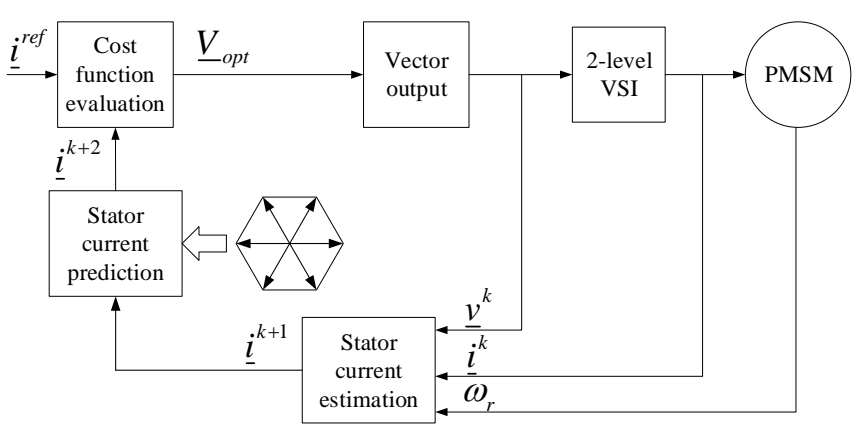

Fig. 2. Diagram of the conventional MBPCC strategy.

$$
\begin{aligned}
& i_{d}^{k+1}=i_{d}^{k}-\frac{R_{s} T_{s}}{L_{d}} i_{d}^{k}+\frac{L_{q} T_{s} \omega_{r}}{L_{d}} i_{q}^{k}+\frac{T_{s}}{L_{d}} v_{d}^{k} \\
& i_{q}^{k+1}=i_{q}^{k}-\frac{R_{s} T_{s}}{L_{q}} i_{q}^{k}-\frac{L_{d} T_{s} \omega_{r}}{L_{q}} i_{d}^{k}+\frac{T_{s}}{L_{q}} v_{q}^{k}-\frac{\varphi_{f} T_{s} \omega_{r}}{L_{q}}
\end{aligned}
$$

where $T_{s}$ is control period; $i_{d}^{k}, i_{q}^{k}$ and $i_{d}^{k+1}, i_{q}^{k+1}$ are the dq-axis currents at $k T_{s}$ and $(k+1) T_{s}$, respectively; $v_{d}^{k}$ and $v_{q}^{k}$ are the input voltage components over $k T_{s}$. It should be noted that there is a one-step delay between the decided voltage vector and the applied vector. Thus, to compensate this inherent delay, the current (3) should first be estimated based on the current measured at the start of $k T_{s}$ and the voltage vector determined in $(k-1) T_{s}$. Then, the current at the start of $(k+2) T_{s}$ can be predicted as

$$
\begin{aligned}
& i_{d}^{k+2}=i_{d}^{k+1}-\frac{R_{s} T_{s}}{L_{d}} i_{d}^{k+1}+\frac{L_{q} T_{s} \omega_{r}}{L_{d}} i_{q}^{k+1}+\frac{T_{s}}{L_{d}} v_{d}^{k+1} \\
& i_{q}^{k+2}=i_{q}^{k+1}-\frac{R_{s} T_{s}}{L_{q}} i_{q}^{k+1}-\frac{L_{d} T_{s} \omega_{r}}{L_{q}} i_{d}^{k+1}+\frac{T_{s}}{L_{q}} v_{q}^{k+1}-\frac{\varphi_{f} T_{s} \omega_{r}}{L_{q}}
\end{aligned}
$$

where $i_{d}^{k+2}, i_{q}^{k+2}$ are the predicted current components at $(k+2) T_{s}, v_{d}^{k+1}$ and $v_{q}^{k+1}$ are the input voltage components in $(k+1) T_{s}$. The stator current (4) can be predicted under all the possible voltage vectors. Finally, the voltage vector that minimizes a predefined cost function (5), which concentrates on the current control performance, will be selected as the optimal one and applied for the next control period.

$$
g=\left|i_{d}^{r e f}-i_{d}^{k+2}\right|+\left|i_{q}^{r e f}-i_{q}^{k+2}\right|
$$

$i_{d}^{r e f}$ and $i_{q}^{r e f}$ are the reference $\mathrm{d}$-axis and q-axis current, respectively. The diagram of the conventional MBPCC is shown in Fig. 2.

\section{Model-Free Predictive CurRent Control}

\section{A. Basic Principle}

In MBPCC, the current estimations (3) and predictions (4) are both parameter dependent. However, the motor parameters may be inaccurate due to measurement error or they may vary due to the changes in operating conditions. Such parameter uncertainties can result in estimated and predicted error, which can further influence the control performance. Therefore, MFPCC, able to avoid the influence of parameter uncertainties by using measured data without system parameters, is introduced. In this strategy, the current estimation can be expressed as

$$
\underline{i}_{j}^{k+1}=\underline{i}^{k}+\Delta \underline{i}_{j}^{k}
$$

where $\underline{i}^{k}=\left[i_{d}^{k}, i_{q}^{k}\right]^{T}$ is the measured stator current vector at $k T_{s}$ and $i_{-j}^{k+1}=\left[i_{d \mid j}^{k+1}, i_{q \mid j}^{k+1}\right]^{T}$ is the estimated current vector at $(k+1) T_{s}$ under the voltage vector applied in $k T_{s}, \underline{V}_{j}^{k}, j \in\{0,1, \ldots, 7\}$, which is determined in the last control period. Because each of the eight voltage vectors results in current gradient on the d-axis and q-axis, which can be measured, all the current gradients can be stored in two LUTs for current estimations and predictions. Thus, $\Delta \underline{i}_{j}^{k}=\left[\Delta \underline{i}_{d \mid j}^{k}, \Delta \underline{i}_{q \mid j}^{k}\right]^{T}$ in (6) denotes the current gradient caused by the $\underline{V}_{j}^{k}$. Then, the current at the start of $(k+2) T_{s}$ can be predicted as

$$
\underline{i}_{j}^{k+2}=\underline{i}_{j}^{k+1}+\Delta \underline{i}_{-j}^{k+1}
$$

where $\underline{i}_{-j}^{k+2}=\left[i_{d \mid j}^{k+2}, i_{q \mid j}^{k+2}\right]^{T}$ is the predicted current at $(k+2) T_{s}$ under the voltage vector applied in $(k+1) T_{s}, \underline{V}_{j}^{k+1}, j \in\{0,1, \ldots, 7\}$, and $\Delta \underline{i}_{j}^{k+1}=\left[\Delta \underline{i}_{d \mid j}^{k+1}, \Delta \underline{i}_{-q \mid j}^{k+1}\right]^{T}$ is the current gradient caused by the $\underline{V}_{j}^{k+1}$.

\section{B.Current Gradient Construction in Conventional Methods}

On the basis of the introduction above, it can be seen that the current gradients under all the candidate voltage vectors are the most important factor for the MFPCC, as their accuracy can directly affect the current estimations and current predictions. However, the current gradient can only be obtained for one voltage vector by measurements in each control period. Thus, the current gradient constructions for the remaining voltage vectors need to be considered.

Fig. 3(a) illustrates the current gradient updating mechanism in [16]. In this strategy, once one of the voltage vectors is applied over a control period, the resulting current gradient is updated in an LUT, whereas the remaining elements are approximated as the old values. Stagnation then occurs with updates to the current gradients under the unapplied voltage vectors. Even worse, if a voltage vector is not applied for many control periods, the long stagnation could even impact the stability of the control system. Thus, a minimum updating frequency is guaranteed in [19] by applying a voltage vector in the next period if it has not been activated during a defined time frame. Nevertheless, because the minimum updating frequency is linked to a predefined time frame, the improvement on the stagnation problem is limited. Moreover, applying a non-optimal voltage vector frequently can result in current ripples and worsen the control performance of the system.

In [20] and [21], to increase the LUT updating frequency, the last three current gradients are used to reconstruct the four under the remaining four voltage vectors, this principle is illustrated in Fig. 3(b). A stringent requirement in this approach, however, is that the last three voltage vectors applied must be different from one another. This means that two different voltage vectors alternately applied for a long interval could corrupt this mechanism, resulting in stagnation. A typical case that often occurs in an MPC is shown in Fig. 4. This requirement can limit 


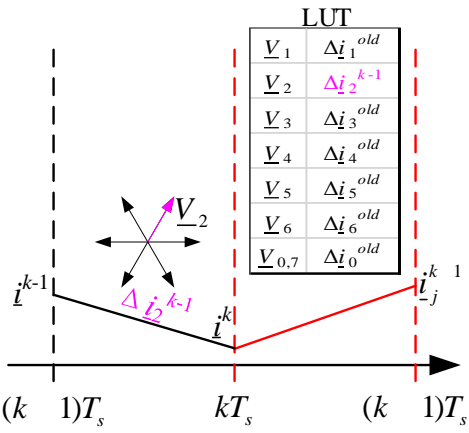

(a)

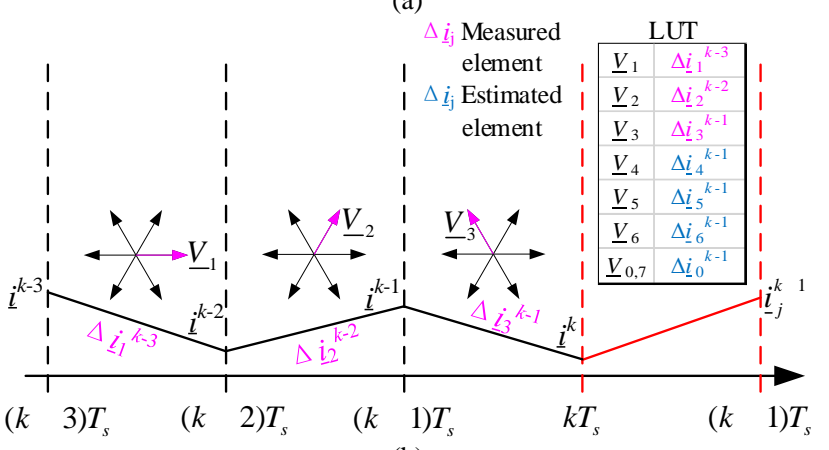

(b)

Fig. 3. Mechanism of current gradient construction in prior methods (a) method in [16] (b) method in [20], [21].

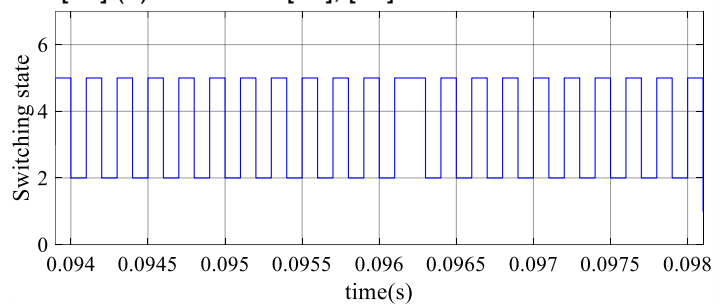

Fig. 4. Two voltage vectors applied for long intervals that can result in stagnation in [20], [21].

the LUT updating frequency of this approach. Furthermore, this approach necessitates identifying all the possible combinations of three-state voltage vectors, which means 210 different vector sequences need to be considered. Thus, to avoid such considerable computations in each control period, a more selective algorithm is required. Even though an identification mechanism that can classify all the vector combinations into six groups is reported in [21], a more simple and intuitive approach to update the current gradients is desirable.

Due to the aforementioned disadvantages, an improved current gradient updating mechanism that uses two succeeding current gradients to estimate the remaining ones is proposed in this paper. This more effective method will be introduced hereafter.

\section{C.Proposed Model-Free Current Gradient Updating Mechanism}

1) Relationship Between Applied Voltage Vector and Current Gradient

In order to provide reliable and accurate current gradients under different voltage vectors, an advanced estimating approach is proposed in this paper, which aims to maximize the LUT updating frequency. To this end, the current gradient due to the applied voltage vector is first investigated.

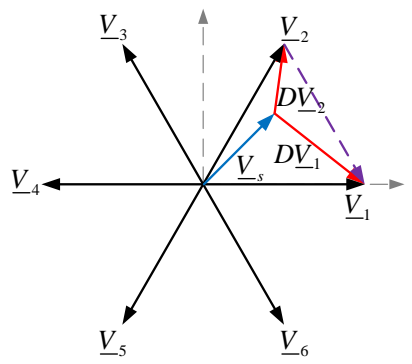

Fig. 5. Principle of voltage deviation.

Because the mechanical time constant $\tau_{m}$ is much larger than the electrical time constants $\tau_{d}=L_{d} / R_{s}$ and $\tau_{q}=L_{q} / R_{s}$, the mechanical speed can be assumed constant over a few time steps and $\omega_{r}=\omega_{r 0}=\Omega$, with $\omega_{r 0}$ being the electrical rotor speed in the steady state. The steady-state model of PMSM can be described as

$$
\begin{aligned}
& V_{s d}=R_{s} I_{s d}-\Omega L_{q} I_{s q} \\
& V_{s q}=R_{s} I_{s q}+\Omega L_{d} I_{s d}+\Omega \varphi_{f}
\end{aligned}
$$

where $V_{s d}$ and $V_{s q}$ are the steady-state voltage components, $I_{s d}$ and $I_{s q}$ are the steady-state current components. By subtracting (8) from (1), a small-signal model of PMSM can be deduced as [22-23]

$$
\begin{aligned}
& \frac{d}{d t} \delta i_{d}=\frac{1}{L_{d}} D v_{d}-\frac{R_{s}}{L_{d}} \delta i_{d}+\Omega \frac{L_{q}}{L_{d}} \delta i_{q} \\
& \frac{d}{d t} \delta i_{q}=\frac{1}{L_{q}} D v_{q}-\frac{R_{s}}{L_{q}} \delta i_{q}-\Omega \frac{L_{d}}{L_{q}} \delta i_{d}
\end{aligned}
$$

where $\delta i_{d}=i_{d}-I_{s d}, \delta i_{q}=i_{q}-I_{s q}$ are small current deviations from the steady state, $D v_{d}=v_{d}-V_{s d}, D v_{q}=v_{q}-V_{s q}$ are voltage deviations from the steady state. Here, the voltage deviations $D v_{d}$ and $D v_{q}$ can be seen as step functions with amplitude $D V_{d}$ and $D V_{q}$, respectively. Based on (9), if one of the basic voltage vectors $\underline{V}_{j}=\left[V_{d \mid j}, V_{q \mid j}\right]^{T}, j \in\{0,1, \ldots, 7\}$, is applied for one control period $T_{s}=\min \left(\tau_{d}, \tau_{q}\right)$, it is shown in [22] that the time gradients of the resulting current deviations $\delta i_{d \mid j}$ and $\delta i_{q \mid j}$ can be approximated as

$$
\begin{aligned}
& \frac{d \delta i_{d \mid j}}{d t}=\frac{D V_{d \mid j}}{L_{d}} \\
& \frac{d \delta i_{q \mid j}}{d t}=\frac{D V_{q \mid j}}{L_{q}}
\end{aligned}
$$

For a control period $T_{s}$, (10) can be written as

$$
\begin{gathered}
\Delta \delta i_{d \mid j}=\frac{T_{s}}{L_{d}} D V_{d \mid j} \\
\Delta \delta i_{q \mid j}=\frac{T_{s}}{L_{q}} D V_{q \mid j}
\end{gathered}
$$

Considering $\quad \Delta \delta i_{d \mid j}=\delta i_{d \mid j}^{t=T_{s}}-\delta i_{d \mid j}^{t=0} \quad, \quad \delta i_{d \mid j}^{t=T_{s}}=i_{d \mid j}^{t=T_{s}}-I_{s d} \quad$, $\delta i_{d \mid j}^{t=0}=i_{d \mid j}^{t=0}-I_{s d}$ and $\Delta \delta i_{q \mid j}=\delta i_{q \mid j}^{t=T_{s}}-\delta i_{q \mid j}^{t=0} \quad, \delta i_{q \mid j}^{t=T_{s}}=i_{q \mid j}^{t=T_{s}}-I_{s q}$, $\delta i_{q \mid j}^{t=0}=i_{q \mid j}^{t=0}-I_{s q},(11)$ can be rewritten as 


$$
\begin{aligned}
\Delta i_{d \mid j} & =\frac{T_{s}}{L_{d}} D V_{d \mid j} \\
\Delta i_{q \mid j} & =\frac{T_{s}}{L_{q}} D V_{q \mid j}
\end{aligned}
$$

where $\Delta i_{d \mid j}=i_{d \mid j}^{t=T_{s}}-i_{d \mid j}^{t=0}, \Delta i_{q \mid j}=i_{q \mid j}^{t=T_{s}}-i_{q \mid j}^{t=0}$ are the dq-axis current gradients over $T_{s}$ due to the applied voltage vector $\underline{V}_{j}$.

It can be seen from (12) that the current gradient is related to the voltage deviation, which is the difference between the voltage vector and the steady-state voltage, as shown in Fig. 5. If the applied voltage vector $\underline{V}_{j}$ is $\underline{V}_{1}$, the voltage deviation $D \underline{V}_{1}$ should be the difference between $\underline{V}_{1}$ and the steady-state voltage $\underline{V}_{s}=\left[V_{s d}, V_{s q}\right]^{T}$. However, estimating $\underline{V}_{s}$ would introduce error that can affect the current gradient estimation in a negative way. Thus, avoiding the use of the steady-state voltage $\underline{V}_{s}$ is desired here. As shown in (8), the steady-state voltage contains the voltage drop on the stator resistor and the back EMF. It is noted that, even though current is fast variable that may change during adjacent control periods, the resulting variation of voltage drop across the resistor is still negligible, compared to the steady-state voltages, $V_{s d}$ and $V_{s q}$. In addition, it has been previously discussed the mechanical speed that related to the back EMF can be assumed as constant over a few time steps. Thus, considering that the control period $T_{s}$ is very short, the steady-state voltage can be considered unchanged during adjacent control periods. Then, a subtraction of two contiguous current gradients caused by two applied voltage vectors can be made as

$$
\begin{aligned}
\Delta i_{d \mid j}^{1}-\Delta i_{d \mid j}^{2} & =\frac{T_{s}}{L_{d}}\left(D V_{d \mid j}^{1}-D V_{d \mid j}^{2}\right) \\
\Delta i_{q \mid j}^{1}-\Delta i_{q \mid j}^{2} & =\frac{T_{s}}{L_{q}}\left(D V_{q \mid j}^{1}-D V_{q \mid j}^{2}\right)
\end{aligned}
$$

where $\Delta i_{-j}^{1}=\left[\Delta i_{d \mid j}^{1}, \Delta i_{q \mid j}^{1}\right]^{T}$ and $\Delta i_{-j}^{2}=\left[\Delta i_{d \mid j}^{2}, \Delta i_{q \mid j}^{2}\right]^{T}$ are the current gradients related to the voltage deviations $D \underline{V}_{j}^{1}=\left[D V_{d \mid j}^{1}, D V_{q \mid j}^{1}\right]^{T}$ and $D \underline{V}_{j}^{2}=\left[D V_{d \mid j}^{2}, D V_{q \mid j}^{2}\right]^{T}$, respectively, the indexes " 1 " and " 2 " represent the variables of the first and second control period. Then, considering $D \underline{V}_{j}^{1}=\underline{V}_{j}^{1}-\underline{V}_{s}$, $D \underline{V}_{j}^{2}=\underline{V}_{j}^{2}-\underline{V}_{s},(13)$ can be rewritten as

$$
\begin{aligned}
& \Delta i_{d \mid j}^{1}-\Delta i_{d \mid j}^{2}=\frac{T_{s}}{L_{d}}\left(V_{d \mid j}^{1}-V_{d \mid j}^{2}\right) \\
& \Delta i_{q \mid j}^{1}-\Delta i_{q \mid j}^{2}=\frac{T_{s}}{L_{q}}\left(V_{q \mid j}^{1}-V_{q \mid j}^{2}\right)
\end{aligned}
$$

Thus, the relationship between the two succeeding current gradients and the two corresponding voltage vectors is derived without the use of the steady-state voltage $\underline{V}_{s}$. It can be seen from Fig. 5 that, if the two applied voltage vectors are $\underline{V}_{1}$ and $\underline{V}_{2}$, the difference between the two voltage vectors can be used instead of the difference between the two voltage deviations, $D \underline{V}_{1}$ and $D \underline{V}_{2}$. For simplicity, (14) can be described in a one-dimensional format as

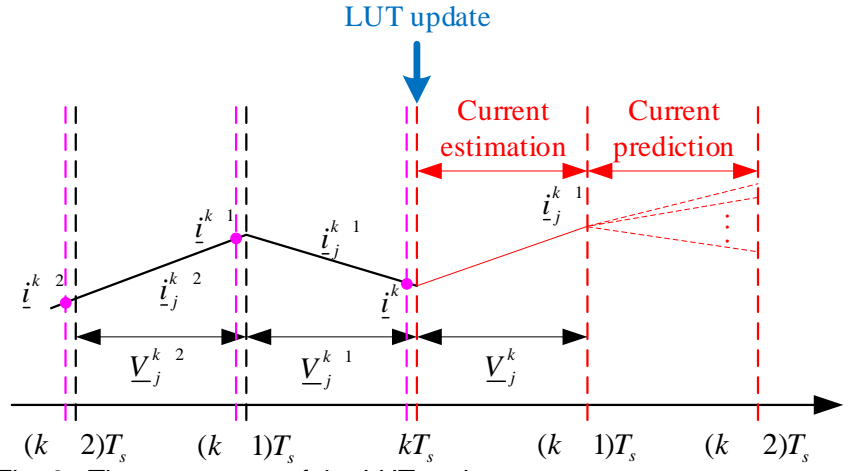

Fig. 6. Time sequence of the LUT update.

$$
\Delta \underline{i}_{j}^{1}-\Delta \underline{i}_{j}^{2}=\frac{T_{s}}{L_{s}}\left(\underline{V}_{j}^{1}-\underline{V}_{j}^{2}\right)
$$

where $\Delta \underline{i}_{j}^{1}=\left[\Delta i_{d \mid j}^{1}, \Delta i_{q \mid j}^{1}\right]^{T}, \Delta \underline{i}_{j}^{2}=\left[\Delta i_{d \mid j}^{2}, \Delta i_{q \mid j}^{2}\right]^{T}$ are the current gradients caused by the two applied voltage vectors $\underline{V}_{j}^{1}=\left[V_{d \mid j}^{1}, V_{q \mid j}^{1}\right]^{T}$ and $\underline{V}_{j}^{2}=\left[V_{d \mid j}^{2}, V_{q \mid j}^{2}\right]^{T}, L_{s}=\left[L_{d}, L_{q}\right]^{T}$.

2) Estimation of Current Gradient for All Voltage Vectors

Based on the analysis above, (15) can be used to estimate the current gradients for all the voltage vectors to be stored in the LUTs. Fig. 6 illustrates the time sequence of the LUT update. At the start of $k T_{s}$, the current gradient over $(k-1) T_{s}$ due to the applied voltage vector $\underline{V}_{j}^{k-1}, j \in\{0,1, \ldots, 7\}$ can be derived based on the current measurements as $\Delta \underline{i}_{j}^{k-1}=\underline{i}^{k}-\underline{i}^{k-1}$. Here, it is noted that the currents are sampled immediately before a new switching state is activated to avoid the current spikes that may occur at the switching instants. Then, with the information from the $(k-2) T_{s},(16)$ can be obtained according to (15).

$$
\Delta_{i_{j}}^{k-1}-\Delta \underline{i}_{-j}^{k-2}=\frac{T_{s}}{L_{s}}\left(\underline{V}_{j}^{k-1}-\underline{V}_{j}^{k-2}\right)
$$

Apart from $\underline{V}_{j}^{k-1}$, if one of the remaining seven voltage vectors $\underline{V}_{j^{\prime}}^{k-1}\left(j^{\prime} \in\{0,1, \ldots, 7\}, j^{\prime} \neq j\right)$, is applied during $(k-1) T_{s}$, a similar relationship can be established as

$$
\Delta \underline{i}_{j^{\prime}}^{k-1}-\Delta \underline{i}_{-j}^{k-2}=\frac{T_{s}}{L_{s}}\left(\underline{V}_{j^{\prime}}^{k-1}-\underline{V}_{j}^{k-2}\right)
$$

Since the control period $T_{s}$ is short enough to assume the motor inductance $L_{s}$ as constant during adjacent control periods, the term $T_{s} / L_{s}$ can be eliminated by combining (16) and (17) as

$$
\frac{\Delta \underline{i}_{j^{\prime}}^{k-1}-\Delta \underline{i}_{j}^{k-2}}{\Delta \underline{i}_{j}^{k-1}-\Delta \underline{i}_{j}^{k-2}}=\frac{\underline{V}_{j^{\prime}}^{k-1}-\underline{V}_{j}^{k-2}}{\underline{V}_{j}^{k-1}-\underline{V}_{j}^{k-2}}
$$

Then, the current gradient under the remaining voltage vector can be estimated as

$$
\Delta \underline{i}_{j^{\prime}}^{k-1}=\frac{\left(\underline{V}_{j^{\prime}}^{k-1}-\underline{V}_{j}^{k-2}\right)\left(\Delta \underline{i}_{j}^{k-1}-\Delta \underline{i}_{j}^{k-2}\right)}{\underline{V}_{j}^{k-1}-\underline{V}_{j}^{k-2}}+\Delta \underline{i}_{-j}^{k-2}
$$

It can be seen that the current gradient estimations are based on measured data only without any reliance on the motor parameters. As the control period $T_{s}$ is very short, the rotor electrical position can be considered unchanged during a few adjacent control periods, the current gradients over $k T_{s}$ and over 


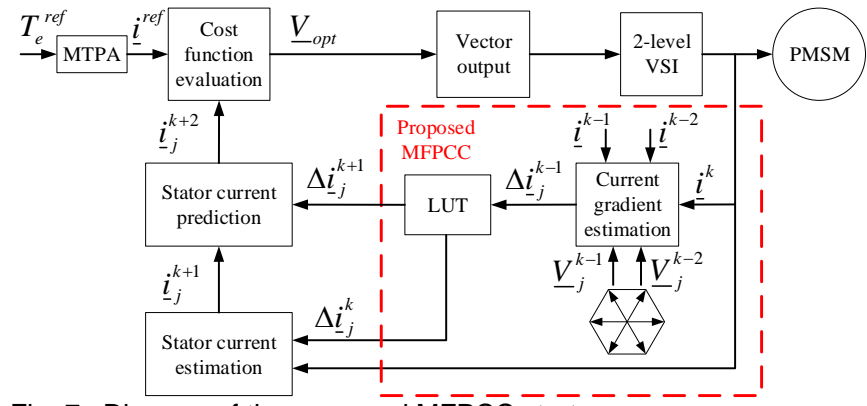

Fig. 7. Diagram of the proposed $\overline{\mathrm{M}} \overline{\mathrm{FPCC}} \overline{\text { strategy. }} \overline{\text {. }}$
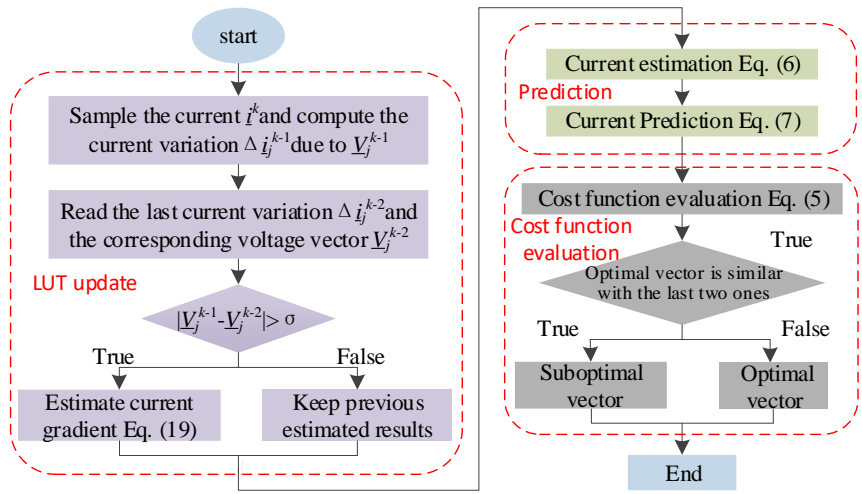

Fig. 8. Flow chart of the proposed MFPCC strategy.

$(k+1) T_{s}$ can be therefore approximated as those over $(k-1) T_{s}$. The current gradients obtained by (19) can be then used in (6) and (7) as $\Delta i_{-j}^{k}$ and $\Delta i_{-j}^{k+1}$ for current estimation and current prediction, respectively.

It should be mentioned that two succeeding similar voltage vectors could lead to inaccurate estimations in (19). Thus, such a situation needs to be well considered. In this paper, a threshold $\sigma$ is introduced. If the denominator of (19) $\underline{V}_{j}^{k-1}-\underline{V}_{j}^{k-2} \geq \sigma$, the current gradients would be estimated by (19). Otherwise, if two succeeding similar vectors occur $\left(\underline{V}_{j}^{k-1}-\underline{V}_{j}^{k-2}<\sigma\right)$, the current gradients would be kept at the previous values. In particular, based on the new outcome of the controller, if more than two succeeding similar vectors would be applied, a suboptimal voltage vector selection could instead be decided upon to avoid the occurrence of several succeeding similar vectors. Otherwise, such a sequence of similar vectors could impede obtaining new accurate current gradient estimations. Such a decision allows for frequent updates to the current gradients, but comes at a cost of operating in a suboptimal manner. Fortunately, a sequence of more than two similar voltage vectors does not occur frequently in an MBPCC or MFPCC. The corresponding influence on the control performance is therefore limited.

Generally, based on the relationship between two succeeding applied voltage vectors and the resulting current gradients, the current gradients under all the vectors can be estimated using the relationships between different voltage vectors. Such an advanced current gradient updating mechanism can effectively solve the stagnation problem in a conventional MFPCC [16] and can also reduce the stagnation caused by applying only two voltage vectors for many periods, which can corrupt the method in [21]. Another improvement on [22] in the proposed approach is the simple and intuitive calculation. The current gradients can

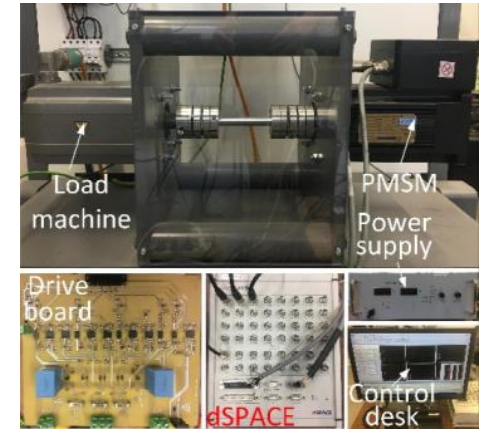

Fig. 9. Experimental setup.

TABLE I

MOTOR PARAMETERS

\begin{tabular}{lcl}
\hline \hline Parameter & Symbol & Value \\
\hline Rated Power & $P_{N}$ & $3.7 \mathrm{~kW}$ \\
Rated torque & $T_{N}$ & $12 \mathrm{Nm}$ \\
Rated speed & $n_{N}$ & $3000 \mathrm{r} / \mathrm{min}$ \\
Number of pole pairs & $p$ & 3 \\
Permanent magnet flux linkage & $\psi_{f}$ & $0.343 \mathrm{~Wb}$ \\
Stator resistance & $R_{s}$ & $0.95 \Omega$ \\
d-axis inductance & $L_{d}$ & $0.0075 \mathrm{H}$ \\
q-axis inductance & $L_{q}$ & $0.018 \mathrm{H}$ \\
Rotor moment of inertia & $J$ & $10.3 \mathrm{kgcm}^{2}$ \\
\hline \hline
\end{tabular}

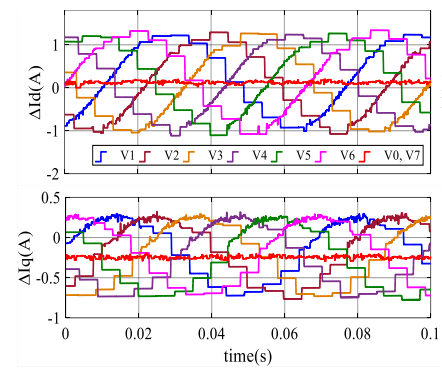

(a)

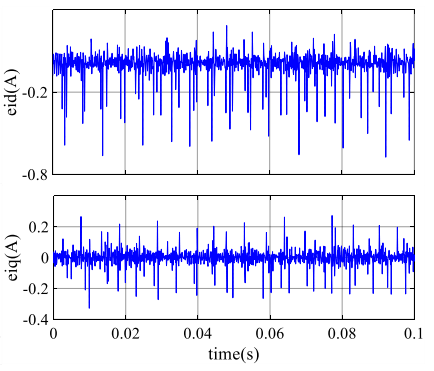

(b)
Fig. 10. Current gradients and prediction errors under the MFPCC-I at $300 \mathrm{r} / \mathrm{min}$ at $8 \mathrm{Nm}$ load. (a) Current gradients under different voltage vectors (b) Current prediction errors.

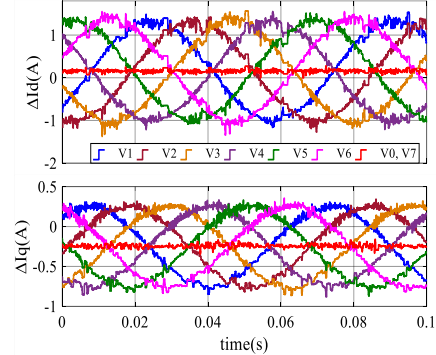

(a)

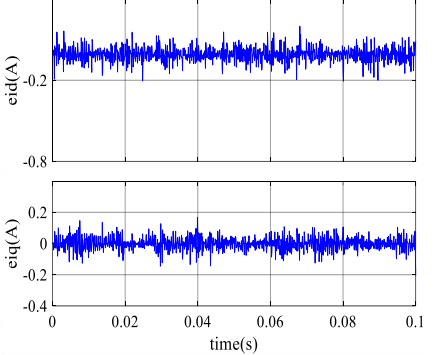

(b)
Fig. 11. Current gradients and prediction errors under the proposed MFPCC at $300 \mathrm{r} / \mathrm{min}$ at $8 \mathrm{Nm}$ load. (a) Current gradients under different voltage vectors (b) Current prediction errors.

TABLE II

STANDARD DEVIATION OF CURRENT PREDICTION ERROR

\begin{tabular}{ccc}
\hline \hline Method & $\begin{array}{c}S_{d} \\
(\mathrm{~A})\end{array}$ & $\begin{array}{c}S_{q} \\
(\mathrm{~A})\end{array}$ \\
\hline MFPCC-I & 0.1102 & 0.0544 \\
Proposed MFPCC & 0.0599 & 0.0423 \\
\hline \hline
\end{tabular}

be estimated based on a simple equation (19), rather than an identification mechanism with an LUT, as in [21].

Fig. 7 and Fig. 8 show the diagram and the flow chart of the proposed MFPCC strategy, respectively. In general, there are three phases to implement the proposed MFPCC. First, the 
current gradients based on all the possible voltage vectors are estimated using the measured currents, thereby refreshing the information in the LUTs. In the second phase, the stator currents under the corresponding voltage vectors in the following control periods are estimated and predicted based on the updated LUTs. Finally, the optimal voltage vector is decided upon according to the results of the cost function evaluation.

\section{SIMULATION AND EXPERIMENTAL RESULTS}

To reveal the validity and practicability of the proposed MFPCC strategy, experiments are carried out on a real PMSM drive setup, as shown in Fig. 9. The parameters of the tested motor are listed in Table I. The control algorithms are implemented using dSPACE Microlabox hardware. To analyze the results, all the measured data are saved and then transferred to Matlab/Simulink to be displayed. In addition, to show the improvements of the proposed MFPCC strategy, the conventional MBPCC and the MFPCC from [19] (referred to as MFPCC-I) are involved for comparison. The control period $T_{s}$ is set at $100 \mu \mathrm{s}$ in all the experimental tests.

\section{A. Experimental Results of Current Gradient Update}

In this section, the current gradients stored in the LUTs under the proposed MFPCC are reported and compared to those under the MFPCC-I when the motor operates at $300 \mathrm{r} / \mathrm{min}$ with an 8 $\mathrm{Nm}$ load. The current gradients due to all the possible voltage vectors under the MFPCC-I are shown in Fig. 10(a). In this strategy, only the current gradient under the applied voltage vector is updated in each control period, whereas the remaining ones are approximated as the old values. Meanwhile, if a voltage vector is not selected in the last contiguous 50 control periods, it will be imposed in the next period to refresh the corresponding current gradient information. Nevertheless, a significant stagnation effect can still be observed in both the d-axis and q-axis currents. Fig. 10(b) shows the predicted current error, which is the difference between the predicted current and the measured current. The unreliable current gradient information leads to large current prediction errors with error spikes, which can further affect the control performance.

On the other hand, the results under the proposed MFPCC are shown in Fig. 11. The proposed strategy aims at updating the current gradients for all the possible voltage vectors in one control period. As a result, a high updating frequency is guaranteed and stagnations are significantly reduced, although some current measurement noises are involved, as can be seen in Fig. 11(a). Here, resistors are used to measure the phase currents. The current noise could be further reduced by using more advanced current sensors. Fig. 11(b) shows the current prediction errors under the proposed MFPCC. As can be seen, reliable current gradient information has been obtained in the proposed MFPCC, such that the proposed MFPCC effectively eliminates the error spikes in the MFPCC-I and reduces the current prediction errors. To statistically compare the results shown in Fig. 10(b) and Fig. 11(b), the standard deviations of the dq-axis current prediction error under the two strategies are calculated using (20), and the results are summarised in Table II.

$$
S=\sqrt{\frac{1}{n} \sum_{i=1}^{n}[e(i)-\mu]^{2}}
$$

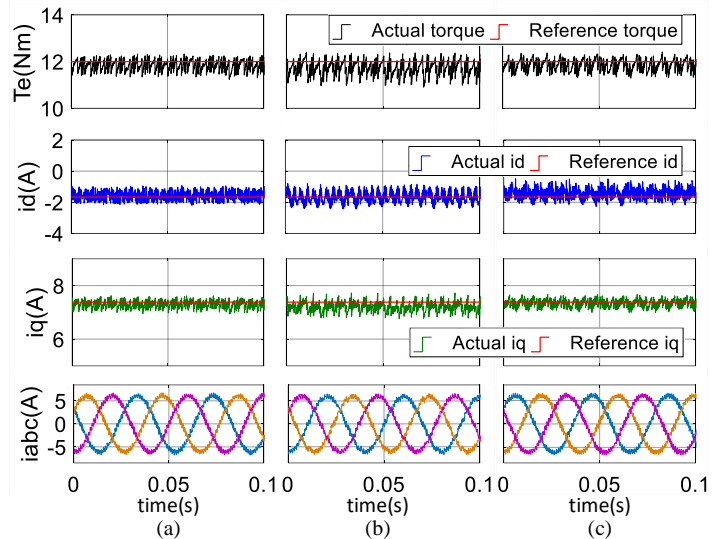

Fig. 12. Experimental results of torque, dq-axis current and phase current under the conventional MBPCC at $500 \mathrm{r} / \mathrm{min}$. (a) With nominal motor parameters (b) With $0.5 L_{d}$ (c) With $0.5 L_{q}$.
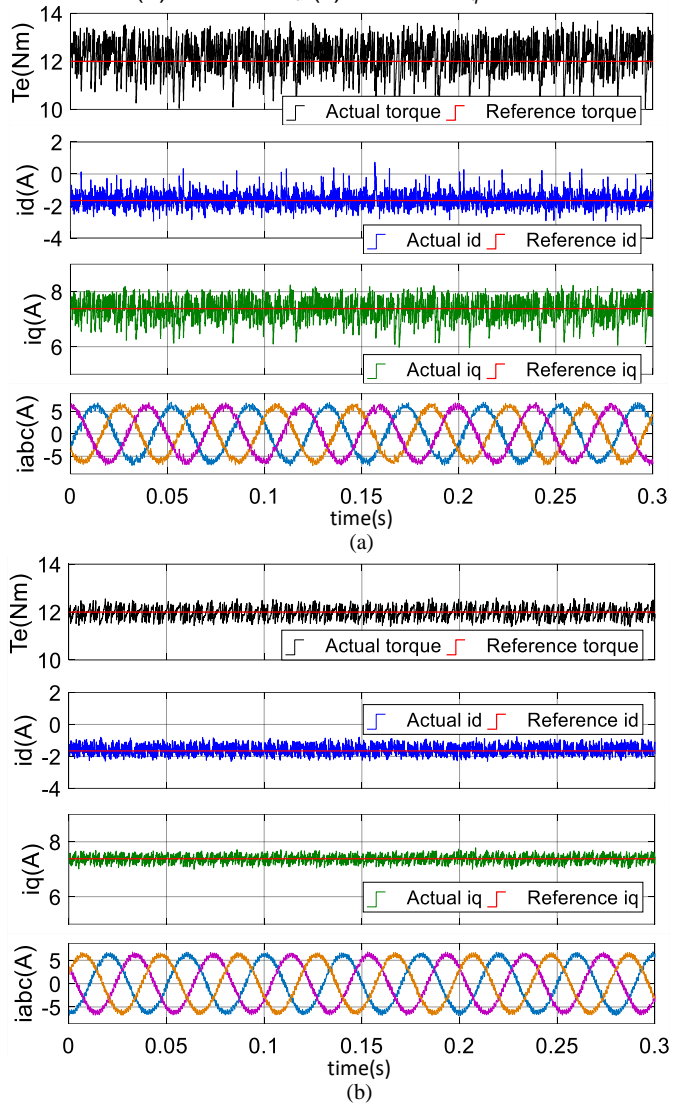

Fig. 13. Experimental results of torque, dq-axis current and phase current under MFPCC strategies at $500 \mathrm{r} / \mathrm{min}$. (a) MFPCC-I (b) Proposed MFPCC.

TABLE III

COMPARISON OF STEADY-STATE PERFORMANCE AT 500 RPM

\begin{tabular}{cccccc}
\hline \hline Method & $\begin{array}{c}T_{e} e^{r i p} \\
(\mathrm{Nm})\end{array}$ & $\begin{array}{c}I_{d}^{r i p} \\
(\mathrm{~A})\end{array}$ & $\begin{array}{c}I_{q}^{r i p} \\
(\mathrm{~A})\end{array}$ & $\begin{array}{c}\text { THD } \\
(\%)\end{array}$ & $\begin{array}{c}f_{a v} \\
(\mathrm{kHz})\end{array}$ \\
\hline MBPCC & 0.277 & 0.282 & 0.162 & 7.53 & 2.81 \\
MBPCC with $0.5 L_{d}$ & 0.409 & 0.332 & 0.246 & 7.76 & 2.87 \\
MBPCC with $0.5 L_{q}$ & 0.267 & 0.415 & 0.137 & 7.46 & 2.78 \\
MFPCC-I & 0.690 & 0.438 & 0.390 & 9.83 & 2.93 \\
Proposed MFPCC & 0.223 & 0.293 & 0.132 & 7.27 & 2.89 \\
\hline \hline
\end{tabular}

$S$ is the standard deviation of the d-axis $\left(S_{d}\right)$ or q-axis $\left(S_{q}\right)$ current prediction error, $n$ is the sampling number, $e$ is the $\mathrm{d}$-axis $\left(e_{i d}\right)$ or q-axis $\left(e_{i q}\right)$ current prediction error, and 


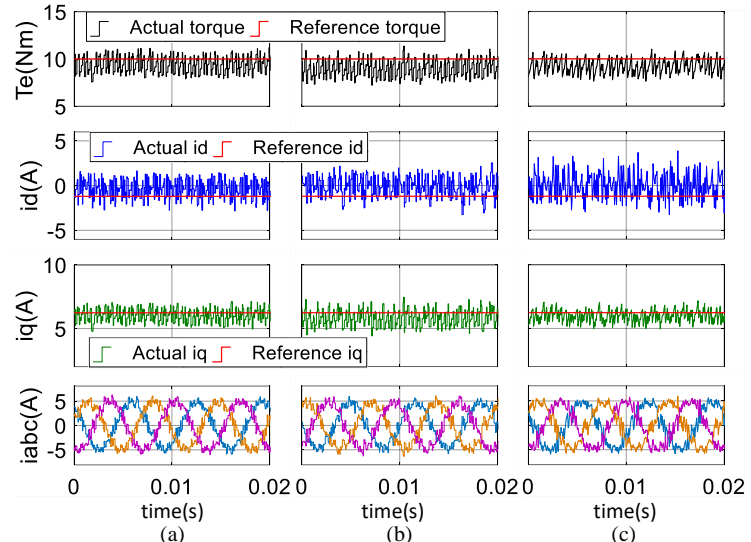

(b)
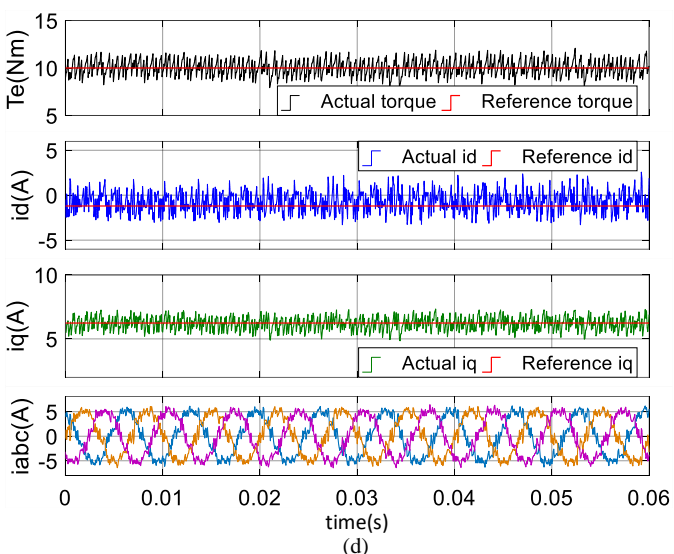

Fig. 14. Experimental results of torque, dq-axis current and phase current at $3000 \mathrm{r} / \mathrm{min}$. (a) MBPCC with nominal motor parameters (b) MBPCC with $0.5 L_{d}$ (c) MBPCC with $0.5 L_{q}$. (d) Proposed MFPCC

TABLE IV

COMPARISON OF STEADY-STATE PERFORMANCE AT 3000 RPM

\begin{tabular}{cccccc}
\hline \hline Method & $\begin{array}{c}T_{e}^{\text {rip }} \\
(\mathrm{Nm})\end{array}$ & $\begin{array}{c}I_{d}^{\text {rip }} \\
(\mathrm{A})\end{array}$ & $\begin{array}{c}I_{q}^{r i p} \\
(\mathrm{~A})\end{array}$ & $\begin{array}{c}\text { THD } \\
(\%)\end{array}$ & $\begin{array}{c}f_{a v} \\
(\mathrm{kHz})\end{array}$ \\
\hline MBPCC & 0.910 & 1.011 & 0.542 & 18.16 & 4.57 \\
MBPCC with $0.5 L_{d}$ & 1.270 & 1.400 & 0.738 & 19.57 & 4.41 \\
MBPCC with $0.5 L_{q}$ & 1.069 & 1.886 & 0.513 & 23.53 & 4.31 \\
Proposed MFPCC & 0.813 & 1.187 & 0.512 & 18.05 & 4.48 \\
\hline \hline
\end{tabular}

$\mu=\frac{1}{n} \sum_{i=1}^{n} e(i)$ is the mean of $e$.

\section{B. Experimental Results of Steady-state performance}

The steady-state performance of the proposed MFPCC strategy is investigated and compared with the conventional MBPCC and the MFPCC-I. As previously mentioned, inductance and PM flux linkage uncertainty has a greater impact on the control performance than resistance uncertainty [12]. Thus, the conventional MBPCC is tested with rated motor parameters, as listed in Table I, and with inductance and PM flux linkage uncertainties. As shown in Fig. 7, the reference stator currents are given by a reference torque based on the maximum torque per ampere (MTPA) principle, using the parameters in Table $I$ in all the tests.

Fig. 12(a) shows the torque, d-axis current, q-axis current and phase currents of the conventional MBPCC with nominal parameters at $500 \mathrm{r} / \mathrm{min}$ with a $12 \mathrm{Nm}$ load. It is noted that the electromagnetic torque is estimated based on measured currents and the torque equation, considering the motor parameters in Table I. The results when the d-axis and q-axis inductance are
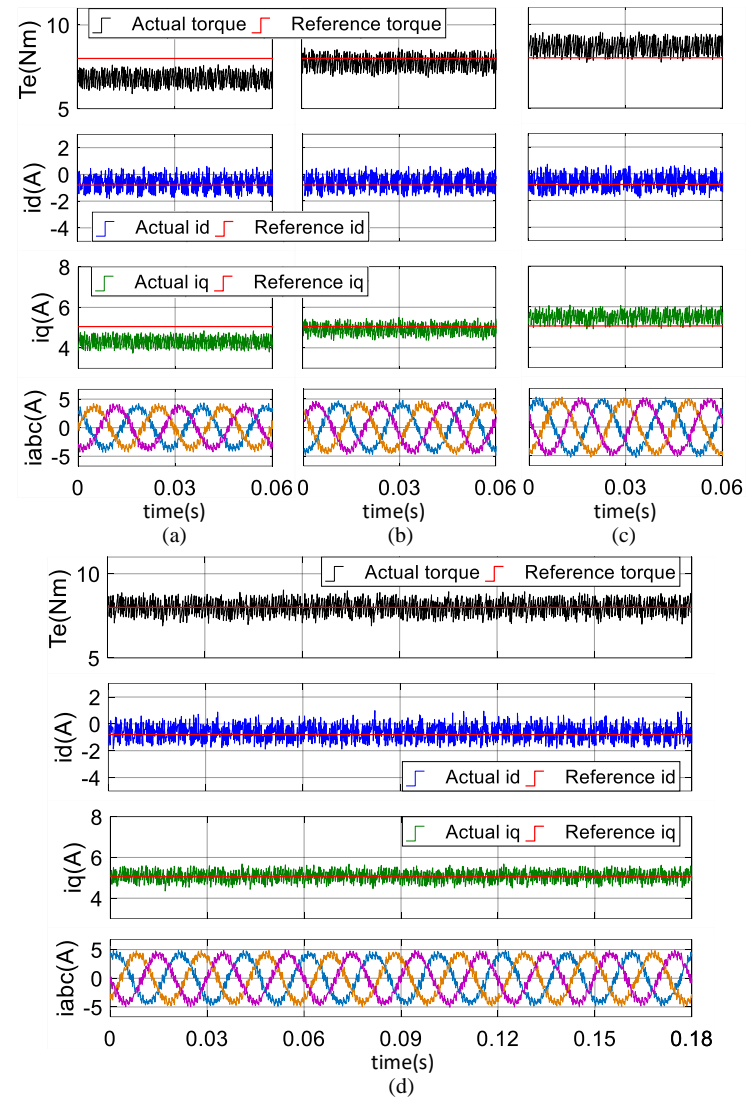

Fig. 15. Experimental results of torque, dq-axis current and phase current at $1000 \mathrm{r} / \mathrm{min}$. (a) MBPCC with $0.5 \varphi_{f}$ (b) MBPCC nominal motor parameters (c) MBPCC with $1.5 \varphi_{f}$. (d) Proposed MFPCC

TABLE V

\begin{tabular}{cccccc}
\multicolumn{6}{c}{ COMPARISON OF STEADY-STATE PERFORMANCE AT 1000 RPM } \\
\hline \hline Method & $\begin{array}{c}T_{e}^{r i p} \\
(\mathrm{Nm})\end{array}$ & $\begin{array}{c}I_{d}^{r i p} \\
(\mathrm{~A})\end{array}$ & $\begin{array}{c}I_{q}^{r i p} \\
(\mathrm{~A})\end{array}$ & $\begin{array}{c}\text { THD } \\
(\%)\end{array}$ & $\begin{array}{c}f_{a v} \\
(\mathrm{kHz})\end{array}$ \\
\hline MBPCC with $0.5 \varphi_{f}$ & 1.238 & 0.506 & 0.762 & 15.49 & 4.67 \\
MBPCC & 0.448 & 0.537 & 0.268 & 13.46 & 4.50 \\
MBPCC with $1.5 \varphi_{f}$ & 0.775 & 0.554 & 0.522 & 12.91 & 4.43 \\
Proposed MFPCC & 0.389 & 0.539 & 0.243 & 13.42 & 4.55 \\
\hline \hline
\end{tabular}

set at $50 \%$ of their nominal values are shown in Fig. 12(b) and Fig. 12(c), respectively. It is evident that the MBPCC is influenced by parameter uncertainties with the parameter mismatches creating current ripples and affecting the steady-state performance.

Although the test presented in Fig. 12(a) is conducted based on the nominal inductance values as listed in Table I, such nominal values may still be inaccurate due to measurement error. Moreover, the actual inductances may vary due to the variable magnetic state of the machine and frequency effects. As a result, even though the nominal inductance values are used in the test shown in Fig. 12(a), they are still not well matched with the actual values, as current deviations from the reference can be observed, especially in the waveform of q-axis current. This further reveals the high parameter sensitivity of the conventional MBPCC.

The experimental results of the MFPCC-I and the proposed MFPCC are shown in Fig. 13. In these strategies, the system model is abandoned. Instead, as shown in (7), current gradients are used to predict future currents without involving any system parameters, thereby totally eliminating the influence of 
parameter mismatches. Nevertheless, as illustrated in Fig. 10, the MFPCC-I strategy suffers the current gradient updating stagnation, which leads to unreliable current predictions, even though a minimum updating frequency is guaranteed. Moreover, as previously mentioned, the minimum updating frequency mechanism imposes a non-optimal voltage vector frequently, which can deteriorate the steady-state performance. The MFPCC-I therefore results in higher current ripples than the conventional MBPCC, which can be seen in Fig. 13(a).

On the other hand, the proposed MFPCC obtains current gradients for each of the voltage vectors in one control period. The stagnation existing in the MFPCC-I is then effectively reduced, and more accurate current predictions are achieved. The current deviations in the conventional MBPCC are therefore eliminated here, and much better steady-state performance is achieved, as shown in Fig. 13(b). In addition, the quantitative results of the three strategies are summarised in Table III, which includes the average torque ripples, $d-q$ axis current ripples, total harmonic distortions (THD) of the phase current and average switching frequencies. Here, the average torque and current ripples are calculated based on (19). The average switching frequency $f_{a v}$ is obtained by collecting the total switching jumps $N$ of the six VSI switches over a test period $t_{N}$ and then calculating $f_{a v}=N / 6 / t_{N}$. From the quantitative results, it is evident that the steady-state performance of the conventional MBPCC is sensitive to parameter mismatches. The proposed MFPCC performs better than the conventional MBPCC with nominal parameters in terms of torque ripple, q-axis current ripple and THD rate at a similar average switching frequency.

$$
x^{r i p}=\sqrt{\frac{1}{n} \sum_{i=1}^{n}\left[x(i)-x^{*}\right]^{2}}
$$

$x^{r i p}$ is the average ripple, $n$ represents the sampling number, $x$ is sampled data, $x^{*}$ is reference value.

The proposed MFPCC is further compared to the conventional MBPCC with different inductance values at 3000 r/min with a $10 \mathrm{Nm}$ load. The results are shown in Fig. 14 and Table IV lists the corresponding quantitative results. As with the previous testing, the conventional MBPCC is sensitive to inductance parameters. Because inductance mismatches exist, ripples and deviations from the reference can be seen with the conventional MBPCC, whereas the proposed MFPCC is not affected by motor parameters and achieves better steady-state performance even at a higher operating speed.

In addition, Fig. 15 reports the performance comparisons of the conventional MBPCC with different PM flux linkage values and the proposed MFPCC at $1000 \mathrm{r} / \mathrm{min}$ with an $8 \mathrm{Nm}$ load. In Fig. 15(a), (b), (c), the PM flux linkage in the conventional MBPCC is set as $50 \%, 100 \%$ and $150 \%$ of the nominal value, respectively. It can be seen that obvious deviations are aroused in the q-axis current and torque by the PM flux linkage mismatches, deteriorating the steady-state performance. In contrast, the proposed MFPCC is not affected by such parameter uncertainty and still obtains good steady-state performance, as can be seen from the statistic results summarized in Table V.
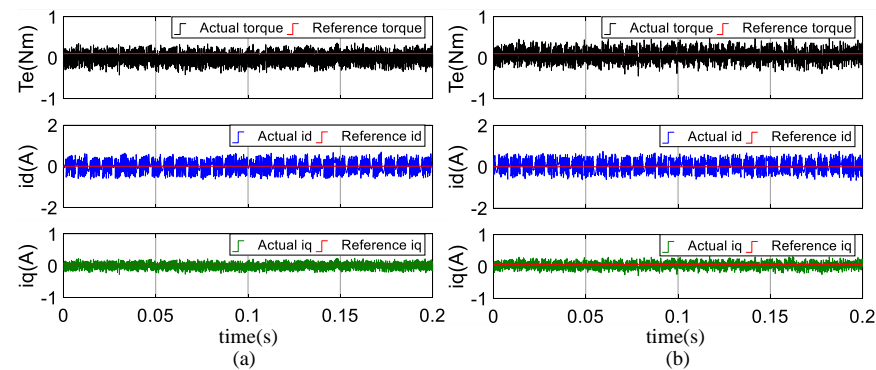

Fig. 16. Experimental results of torque and dq-axis current at 500 r/min, $0.1 \mathrm{Nm}$ load. (a) Conventional MBPCC (b) proposed MFPCC.

TABLE VI

QUANTITATIVE RESULTS OF LOW LOAD TEST

\begin{tabular}{ccccc}
\hline \hline Method & $\begin{array}{c}T_{e}^{r i p} \\
(\mathrm{Nm})\end{array}$ & $\begin{array}{c}I_{d}^{\text {rip }} \\
(\mathrm{A})\end{array}$ & $\begin{array}{c}I_{q}^{r i p} \\
(\mathrm{~A})\end{array}$ & $\begin{array}{c}f_{a v} \\
(\mathrm{kHz})\end{array}$ \\
\hline Conventional MBPCC & 0.180 & 0.258 & 0.117 & 5.57 \\
Proposed MFPCC & 0.161 & 0.270 & 0.105 & 5.46 \\
\hline \hline
\end{tabular}

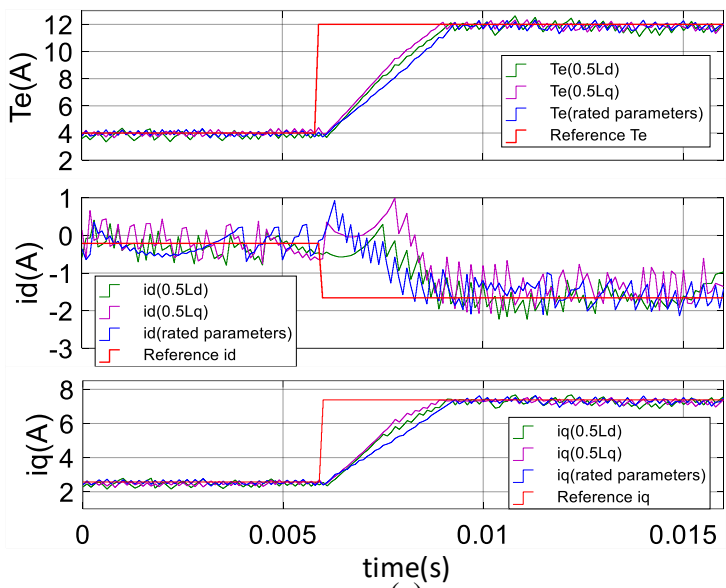

(a)
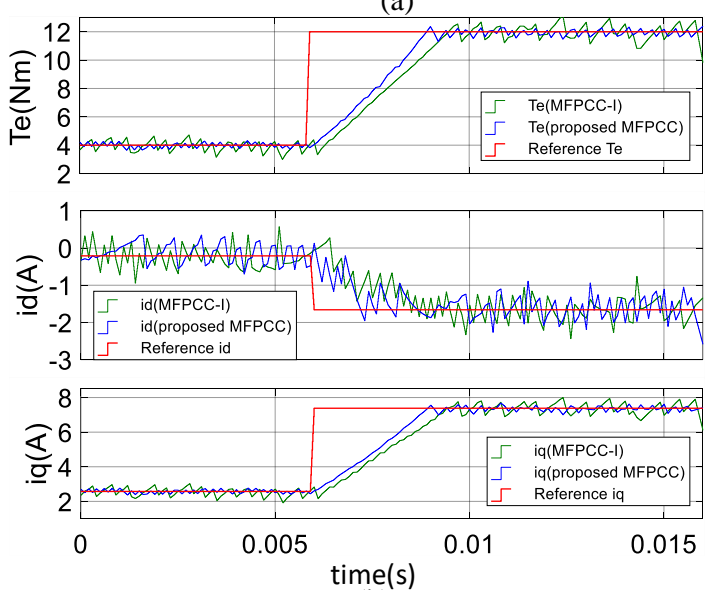

(b)

Fig. 17. Dynamic performance comparison when load changing. (a) Conventional MBPCC (b) MFPCC-I and proposed MFPCC.

\section{Experimental Results of Low Load Test}

Additionally, a low load test is conducted in which the conventional MBPCC with nominal parameters and the proposed MFPCC are compared at $500 \mathrm{r} / \mathrm{min}$ with a $0.1 \mathrm{Nm}$ load. Fig. 16 shows the results in terms of torque and dq-axis current. In this case, even though the reference currents are almost zero, the proposed MFPCC can still obtain enough information from the current variations to operate normally. The quantitative results of the torque ripple, current ripple and 


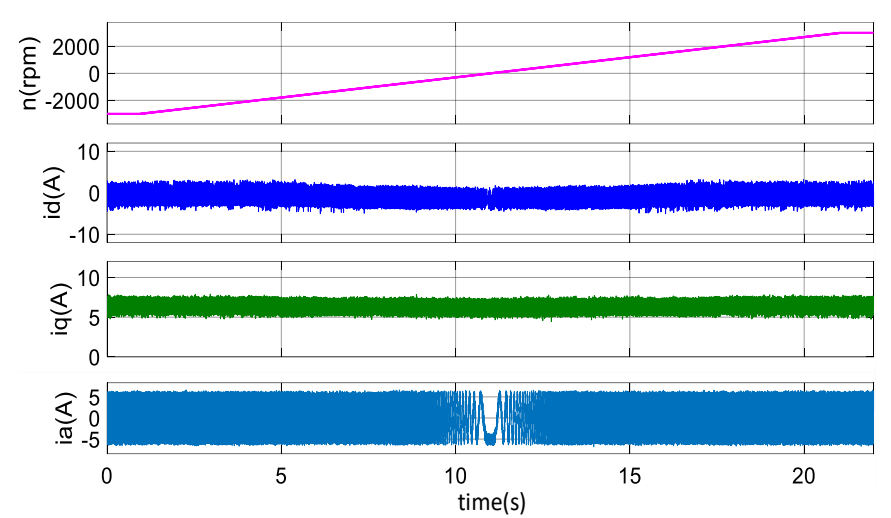

Fig. 18. Speed reversal test of the proposed MFPCC.

TABLE VII

COMPUTATION BURDEN COMPARISON

\begin{tabular}{cccc}
\hline \hline Task & $\begin{array}{c}\text { Conventional } \\
\text { MBPCC }\end{array}$ & MFPCC-I & $\begin{array}{c}\text { Proposed } \\
\text { MFPCC }\end{array}$ \\
\hline \hline LUT update & $0 \mu \mathrm{s}$ & $1.4 \mu \mathrm{s}$ & $5.1 \mu \mathrm{s}$ \\
Prediction & $11.7 \mu \mathrm{s}$ & $11.2 \mu \mathrm{s}$ & $11.2 \mu \mathrm{s}$ \\
Evaluation & $7.5 \mu \mathrm{s}$ & $7.5 \mu \mathrm{s}$ & $7.5 \mu \mathrm{s}$ \\
Total time & $19.2 \mu \mathrm{s}$ & $20.1 \mu \mathrm{s}$ & $23.8 \mu \mathrm{s}$ \\
\hline \hline
\end{tabular}

average switching frequency under the two strategies are summarised in Table VI. Compared to the conventional MBPCC, the proposed MFPCC performs better in terms of torque ripple and $\mathrm{q}$-axis current ripple at a similar average switching frequency.

\section{Experimental Results of Torque Step Test}

To show the dynamic performance of the three strategies, comparative tests are carried out where the load suddenly changed from $4 \mathrm{Nm}$ to $12 \mathrm{Nm}$. Fig. 17(a) shows the transient torque and d-q axis currents under the conventional MBPCC. The blue lines are the results with nominal motor parameters, whereas the green and purple lines are the results under inductance mismatches. The comparative results under the two MFPCC strategies are shown in Fig. 17(b). There are overshoots in the waveforms of the d-axis currents under the conventional MBPCC when the reference changes. Conversely, the two MFPCC strategies complete this transition and enter the steady state without overshoots.

It is noted that the information about current gradients with the MFPCC-I can be unreliable due to the stagnation problem. Such unreliable information can lead the cost function to make a bad and incorrect decision when determining a voltage vector to be applied. This phenomenon could occur in both steady state and dynamic state, thus affecting both steady-state performance and dynamic response. In contrast, the current gradients with the proposed MFPCC are up to date and reliable due to the proposed updating mechanism. This guarantees the cost function to make a good decision in steady state as well as dynamic state. As a result, it can be seen in Fig. 17(b) that the proposed MFPCC achieves a faster response in the torque and the $\mathrm{q}$-axis current step compared to the MFPCC-I, demonstrating the good dynamic performance of the proposed MFPCC strategy.

\section{E. Experimental Results of Speed Reversal Test}

To further confirm the dynamic performance of the proposed MFPCC, a speed reversal test is carried out in which the rotor speed reversed from $-3000 \mathrm{r} / \mathrm{min}$ to $3000 \mathrm{r} / \mathrm{min}$ with a $10 \mathrm{Nm}$

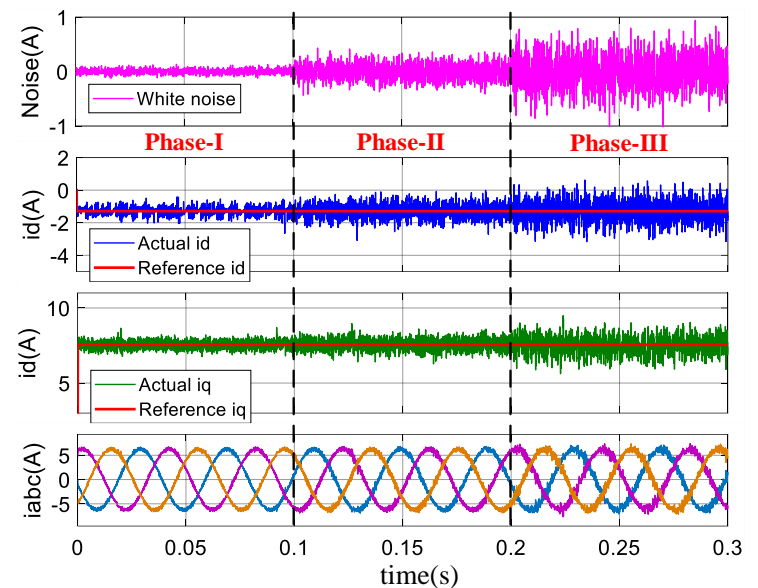

Fig. 19. Simulation results of steady-state performance considering current noise.

load. Fig. 18 shows the waveforms of rotor speed, dq-axis currents and phase current. It should be noted that the speed and torque are set separately. The reference torque is set externally, whereas the speed of the PMSM is controlled by the load machine, a servo motor driven by the Siemens SINAMICS drive system. During the speed changing process, the reference torque and reference dq-axis currents are therefore not affected and are kept constant, as shown in Fig. 18. The results reveal that the proposed MFPCC performs well across the entire speed range.

\section{F. Experimental Results of Computational Time}

The computational time is compared for the conventional MBPCC, the MFPCC-I and the proposed MFPCC. The results for the three strategies are shown in Table VII. In the conventional MBPCC, the currents need to be predicted under seven voltage vectors, an algorithm that takes $19.2 \mu \mathrm{s}$ to conduct. In the MFPCC-I, except for the current predictions and cost function evaluations, the current gradients due to the applied voltage vector are measured and used to update one of the corresponding LUT elements, and the computational time slightly increases to $20.1 \mu \mathrm{s}$. In the proposed MFPCC, all the LUT elements are updated based on a simple equation (19), and the computational time of the algorithm is $23.8 \mu \mathrm{s}$. Generally, the increased computational time of the proposed MFPCC is caused by the additional LUT update operation. However, considering the improved control performance, such slight increase in computational time is quite acceptable for the proposed MFPCC.

\section{G. Simulation Results of Stability Test}

In the proposed MFPCC, the current gradients are obtained based on current measurements. Thus, the measurement noise in current can be inevitably involved. To confirm the stability of the proposed MFPCC against current noise, a simulation considering different levels of current noise is carried out. Here, white noise is added to the dq-axis currents that are further used in the proposed MFPCC. Fig. 19 shows the steady-state performance considering the white noise. From top to bottom, the waveforms are the white noise added to the dq-axis currents, d-axis current, q-axis current and phase currents. To clearly show how current noise can influence the proposed MFPCC, the variance of the white noise in the Phase-I, Phase-II and Phase-III is set as $0.002 \mathrm{~A}$ and $0.02 \mathrm{~A}$ and $0.1 \mathrm{~A}$, 
respectively. In the Phase-III, the amplitude of the peak noise is up to $1 \mathrm{~A}$. It can be seen that even though the current noise increases, the proposed MFPCC still performs stably, although the steady-state performance is influenced by the increased noise. The stability of the proposed MFPCC is then further confirmed. Moreover, the effect of current noise could be limited by a more accurate current sensing [21].

\section{CONCLUSION}

An improved model-free predictive current control is proposed in this paper. In contrast to the model-based predictive control, which is sensitive to parameter mismatches, the proposed MFPCC does not depend on any motor parameters, therefore avoiding the influence of parameter mismatches. The relationship between two succeeding applied voltage vectors and the resulting current gradients is exploited to deduce the current gradients for all the possible voltage vectors within one control period. The main benefit is that such high frequency updating of the LUTs provides a reliable foundation for the current estimations and predictions.

The proposed MFPCC strategy, with simple computations, does not involve any complicated operating mechanisms. This could contribute to its development for industrial applications. Due to the feature of parameter independence, the proposed MFPCC could be useful in cases where motor knowledge is not adequately provided. The validity of the proposed strategy has been demonstrated by experiments comparing the conventional MBPCC to the previous MFPCC. The results have revealed the proposed MFPCC can obtain similar performance in comparison with the conventional MBPCC incorporating nominal parameters without reliance on system model. In addition, the proposed MFPCC achieves significant performance improvements compared to the previous MFPCC.

\section{REFERENCES}

[1] Kang, S.-W., Soh, J.-H., and Kim, R.-Y., "Symmetrical Three-Vector-Based Model Predictive Control with Deadbeat Solution for Ipmsm in Rotating Reference Frame", IEEE Trans. Ind. Electron., 2020, 67, (1), pp. 159-168.

[2] Guazzelli, P.R.U., de Andrade Pereira, W.C., de Oliveira, C.M.R., de Castro, A.G., and de Aguiar, M.L., "Weighting Factors Optimization of Predictive Torque Control of Induction Motor by Multiobjective Genetic Algorithm", IEEE Trans. Power Electron., 2019, 34, (7), pp. 6628-6638.

[3] Xue, Y., Meng, D., Yin, S., Han, W., Yan, X., Shu, Z., and Diao, L., "Vector-Based Model Predictive Hysteresis Current Control for Asynchronous Motor", IEEE Trans. Ind. Electron., 2019, 66, (11), pp. 8703-8712.

[4] Wang, F., Xie, H., Chen, Q., Davari, S.A., Rodriguez, J., and Kennel, R., "Parallel Predictive Torque Control for Induction Machines without Weighting Factors", IEEE Trans. Power Electron., 2020, 35, (2), pp. 1779-1788.

[5] Andersson, A. and Thiringer, T., "Assessment of an Improved Finite Control Set Model Predictive Current Controller for Automotive Propulsion Applications", IEEE Trans. Ind. Electron., 2020, 67, (1), pp. 91-100.

[6] Yan, L., Wang, F., Dou, M., Zhang, Z., Kennel, R., and Rodriguez, J., "Active Disturbance-Rejection-Based Speed Control in Model Predictive Control for Induction Machines," IEEE Trans. Ind. Electron., 2020, 67, (4), pp. 2574-2584.

[7] Siami, M., Khaburi, D.A., and Rodriguez, J., "Torque Ripple Reduction of Predictive Torque Control for Pmsm Drives with Parameter Mismatch", IEEE Trans. Power Electron., 2017, 32, (9), pp. 7160-7168.

[8] Y. Zhang and H. Yang, "Generalized Two-Vector-Based Model-Predictive Torque Control of Induction Motor Drives," IEEE Trans. Power Electron., vol. 30, no. 7, pp. 3818-3829, 2015.
[9] Y. Zhang and H. Yang, "Two-Vector-Based Model Predictive Torque Control Without Weighting Factors for Induction Motor Drives," IEEE Trans. Power Electron., vol. 31, no. 2, pp. 1381-1390, 2016.

[10] Z. Zhou, C. Xia, Y. Yan, Z. Wang, and T. Shi, "Torque Ripple Minimizatin of Predictive Torque Control for PMSM With Extended Control Set," IEEE Trans. Ind. Electron., vol. 64, no. 9, pp. 6930-6939, 2017.

[11] M. Xio, T. Shi, Y. Yan, W. Xu, and C. Xia, "Predictive Torque Control of Permannt Magnet Synchronous Motors Using Flux Vector," IEEE Trans. Ind Appl., vol. 54, no. 5, pp. 4437-4446, 2018.

[12] Zhang, X., Zhang, L., and Zhang, Y., "Model Predictive Current Control for Pmsm Drives with Parameter Robustness Improvement", IEEE Trans. Power Electron., 2019, 34, (2), pp. 1645-1657.

[13] Chen, Z., Qiu, J., and Jin, M., "Adaptive Finite-Control-Set Model Predictive Current Control for Ipmsm Drives with Inductance Variation", IET Electr. Power Appl., 2017, 11, (5), pp. 874-884.

[14] Yang, M., Lang, X., Long, J., and Xu, D., "Flux Immunity Robust Predictive Current Control with Incremental Model and Extended State Observer for Pmsm Drive", IEEE Trans. Power Electron., 2017, 32, (12), pp. 9267-9279.

[15] X. Liu, L. Zhou, J. Wang, X. Gao, Z. Li, and Z. Zhang, "Robust Predictive Current Control of Permanent-Magnet Synchronous Motors With Newly Designed Cost Function," IEEE Trans. Power Electron., vol. 35, no. 10, pp. 10778-10788, 2020.

[16] Lin, C.-K., Liu, T.-H., Yu, J.-t., Fu, L.-C., and Hsiao, C.-F., "Model-Free Predictive Current Control for Interior Permanent-Magnet Synchronous Motor Drives Based on Current Difference Detection Technique", IEEE Trans. Ind. Electron., 2014, 61, (2), pp. 667-681.

[17] N. Chaovalit, and S. Yamamoto, "Model-Free Predictive Control and Its Relation to Parameter-Estimation-Based Predictive Control", Proc. Int. Symp. Control Syst., July 2019, pp. 84-89.

[18] Siami, M., Khaburi, D.A., Abbaszadeh, A., and Rodriguez, J., "Robustness Improvement of Predictive Current Control Using Prediction Error Correction for Permanent-Magnet Synchronous Machines", IEEE Trans. Ind. Electron., 2016, 63, (6), pp. 3458-3466.

[19] Lin, C.-K., Yu, J.-t., Lai, Y.-S., and Yu, H.-C., "Improved Model-Free Predictive Current Control for Synchronous Reluctance Motor Drives", IEEE Trans. Ind. Electron., 2016, 63, (6), pp. 3942-3953.

[20] D. Da Rú, M. Polato, and S. Bolognani, "Model-free predictive current control for a SynRMdrive based on an effective update of measured current responses," in Proc. IEEE Int. Symp. Predictive Control Electr. Drives Power Electron., Sep. 2017, pp. 119-124.

[21] Carlet, P.G., Tinazzi, F., Bolognani, S., and Zigliotto, M., “An Effective Model-Free Predictive Current Control for Synchronous Reluctance Motor Drives", IEEE Trans. Ind. Appl., 2019, 55, (4), pp. 3781-3790.

[22] De Belie, F.M.L., Sergeant, P., and Melkebeek, J.A., "A Sensorless Drive by Applying Test Pulses without Affecting the Average-Current Samples", IEEE Trans. Power Electron., 2010, 25, (4), pp. 875-888.

[23] H. Li, F. D. Belie and J. Melkebeek, "Introducing a pulse response based rotor position estimator for DTC IPMSM drives," In Proc. IEEE International Symposium on Sensorless Control for Electrical Drives, Catania, Italy, Sep. 2017, pp. 133-138.

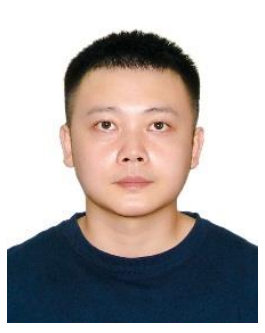

Chenwei Ma was born in China in 1990. He received the B.S. degree in automation and the M.S degree in electric machine and electric apparatus from the Harbin University of Science and Technology, Harbin, China, in 2012 and 2015, respectively. He is currently working toward the Ph.D. degree with the College of Intelligent Systems Science and Engineering the College of Automation, Harbin Engineering University, Harbin, China and with the Department of Electrical Energy, Metals, Mechanical Constructions \& Systems, Ghent University, Ghent,

Belgium. control. 


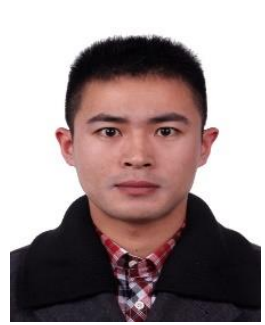

Huayu Li was born in Hunan Province, China, in 1989. He received the Bachelor's degree in automation from Central South University, Changsha, China in 2012 and the Master's degree in electrical engineering from Naval University of Engineering, Wuhan, China in 2014. At present, he is with the Electrical Energy Laboratory, Ghent University, Ghent, Belgium, where he is currently working toward the Ph.D. degree in electromechanical engineering.

His current research interests include sensorless control, machine parameters identification, direct torque control and model predictive control.

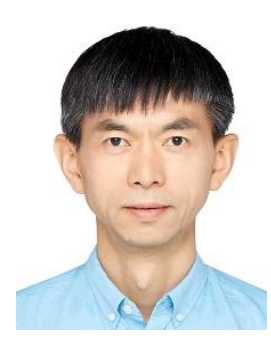

Xuliang Yao received the Ph.D. degree from the College of Automation, Harbin Engineering University, Harbin, China, in 2005, where he is currently a Professor.

His current research interests include power electronics and power drive, ship electric propulsion, and control theory of shipping motion.

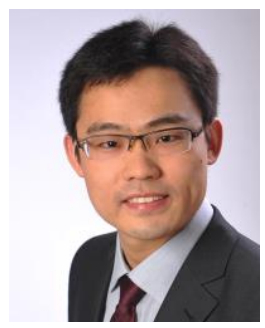

Zhenbin Zhang (S'11-M'14-SM'18), was born in Shandong, China, in 1984. He received the Ph.D. degree at the Institute for Electrical Drive Systems and Power Electronics (EAL), Technical University of Munich (TUM), Germany, with "summa cum laude". From 2016 to 2017, he worked as a Research Fellow and the group-leader for "Modern control strategies for electrical drives" group in EAL of TUM. Since 2017, he has been a full professor and lab.-director at Shandong Uni., Jinan, China. Since 2020, he has been appointed as the director of International Center for Intelligent Energy and Power Conversion Systems (IEPCS) at Shandong Uni.

Dr. Zhang received the VDE-Award, Suedbayern, Germany, in 2017. He is an Associate Editor for IEEE Trans. Ind. Electron. His research interests include power electronics and electrical drives, sustainable energy systems, smart- and micro-grid.

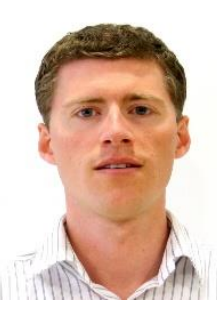

Frederik De Belie was born in Belgium in 1979. He received the MSc degree in electromechanical engineering, in 2002, and the $\mathrm{PhD}$ degree in electromechanical engineering, in 2010, both from Ghent University, Ghent, Belgium. For his PhD work and patent on the seamless integration of sensorless control in drives he was awarded in 2010 the Iwan Akerman Award, awarded on the initiative of Atlas Copco Airpower and Research Foundation Flanders. $\mathrm{He}$ is currently an Associate Professor with the Department of Electromechanical, Systems and Metal Engineering, Ghent University, where he teaches bachelor and master courses in electric drives.

His current research interests include identification, monitoring, dynamical modeling and smart control theory applied to power converters and electrical drives. 